University of Nebraska - Lincoln

DigitalCommons@University of Nebraska - Lincoln

Faculty Publications from the Department of Engineering Mechanics

Mechanical \& Materials Engineering,

Department of

2008

\title{
Rippling of Polymer Nanofibers
}

\author{
Xiang-Fa Wu \\ Department of Engineering Mechanics, University of Nebraska-Lincoln, xfwu@unlserve.unl.edu \\ Yulia Kostogorova-Beller \\ University of Nebraska - Lincoln \\ Alexander Goponenko \\ University of Nebraska - Lincoln, agoponenko2@unl.edu \\ Haoqing $\mathrm{Hou}$ \\ Jiangxi Normal University, Nanchang 330022, China \\ Yuris A. Dzenis \\ University of Nebraska-Lincoln, ydzenis@unl.edu
}

Follow this and additional works at: https://digitalcommons.unl.edu/engineeringmechanicsfacpub

Part of the Mechanical Engineering Commons

Wu, Xiang-Fa; Kostogorova-Beller, Yulia; Goponenko, Alexander; Hou, Haoqing; and Dzenis, Yuris A., "Rippling of Polymer Nanofibers" (2008). Faculty Publications from the Department of Engineering Mechanics. 44.

https://digitalcommons.unl.edu/engineeringmechanicsfacpub/44

This Article is brought to you for free and open access by the Mechanical \& Materials Engineering, Department of at DigitalCommons@University of Nebraska - Lincoln. It has been accepted for inclusion in Faculty Publications from the Department of Engineering Mechanics by an authorized administrator of DigitalCommons@University of Nebraska - Lincoln. 


\title{
Rippling of polymer nanofibers
}

\author{
Xiang-Fa Wu, ${ }^{1,2, *}$ Yulia Y. Kostogorova-Beller, ${ }^{1}$ Alexander V. Goponenko, ${ }^{1}$ Haoqing Hou, ${ }^{3}$ and Yuris A. Dzenis ${ }^{1}$ \\ ${ }^{1}$ Department of Engineering Mechanics, Nebraska Center for Materials and Nanoscience, University of Nebraska-Lincoln, \\ Lincoln, Nebraska 68588-0526, USA \\ ${ }^{2}$ Department of Mechanical Engineering and Applied Mechanics, North Dakota State University, Dolve Hall 111, \\ Fargo, North Dakota 58108-6050, USA \\ ${ }^{3}$ Chemistry and Chemical Engineering College, Jiangxi Normal University, Nanchang 330022, China \\ (Received 27 March 2008; revised manuscript received 25 September 2008; published 31 December 2008)
}

\begin{abstract}
This paper studies the evolution mechanism of surface rippling in polymer nanofibers under axial stretching. This rippling phenomenon has been detected in as-electrospun polyacrylonitrile in recent single-fiber tension tests, and in electrospun polyimide nanofibers after imidization. We herein propose a one-dimensional nonlinear elastic model that takes into account the combined effect of surface tension and nonlinear elasticity during the rippling initiation and its evolution in compliant polymer nanofibers. The polymer nanofiber is modeled as an incompressible, isotropically hyperelastic Mooney-Rivlin solid. The fiber geometry prior to rippling is considered as a long circular cylinder. The governing equation of surface rippling is established through linear perturbation of the static equilibrium state of the nanofiber subjected to finite axial prestretching. The critical stretch and ripple wavelength are determined in terms of surface tension, elastic property, and fiber radius. Numerical examples are demonstrated to examine these dependencies. In addition, a critical fiber radius is determined, below which the polymer nanofibers are intrinsically unstable. The present model, therefore, is capable of predicting the rippling condition in compliant nanofibers, and can be further used as a continuum mechanics approach for the study of surface instability and nonlinear wave propagation in compliant fibers and wires at the nanoscale.
\end{abstract}

DOI: 10.1103/PhysRevE.78.061804

PACS number(s): 61.41.+e, 82.35.-x, 46.25.Cc

\section{INTRODUCTION}

Ultrathin polymer fibers with diameter ranging from $1 \mathrm{~nm}$ to several micrometers have been produced extensively by the electrospinning technique [1,2]. Due to their high surface area to volume ratio, high tensile strength, controllable diameter, surface morphology, and microstructure, as well as low processing cost, electrospun nanofibers are finding rapidly increasing applications as a novel class of one-dimensional (1D) nanomaterials, which include protective clothing [3,4], fine filtration $[5,6]$, nanocomposites [7], templates for producing metallic nanowires and carbon nanotubes $[8,9]$, precursors for synthesizing carbon nanofibers $[10,11]$, biomedical engineering and technologies (e.g., scaffolds for tissue growth [12,13] and drug delivery systems [14]), nanosensors and nanoelectromechanical systems $[15,16]$, and microdevices [17], among others. Continuous nanofibers in an electrospinning process can be collected in the form of porous nonwoven mats or aligned nanofibrous films with the aid of auxiliary electrical fields [18]. To date, over 200 synthetic and natural polymers have been electrospun successfully into continuous nanofibers. The number of research publications on electrospinning techniques and the resulting fibers has doubled annually in recent years. In addition, several popular review articles have been dedicated to the research progress in nanofiber manufacturing, mechanical characterization, alignment techniques, and potential applications in broad fields [14,19-24].

\footnotetext{
*Author to whom correspondence should be addressed. FAX: 1+701-231-8913. Xiangfa.Wu@ndsu.edu
}

As in conventional bulk structural and functional materials, the mechanical properties of nanofibers (e.g., modulus, tensile strength, yielding property, etc.) are fundamental to their environmental response and expected functionalities when nanofibers are integrated into nanocomposites and microstructural components. Nanofibers with high strength and high toughness are always desired in view of their end use [7]. In addition, fibrous materials at the nanoscale may exhibit unique mechanical behaviors that are essentially different from those of their bulk counterparts. Such unique properties may further influence the deformation, dynamics, stability, adhesion, contact, friction, wetting, and global mechanical response of the resulting nanofilamentary materials and nanodevices [25-30]. In recent years, substantial effort has been devoted to understanding the mechanical behavior of individual nanofibers fabricated by electrospinning [31-38]. Among these, atomic force microscope (AFM) serves as a typical instrument and has been employed by several research groups for the mechanical characterization (e.g., axial modulus determination) of electrospun nanofibers [32-35]. To date, three AFM-based testing methods have been developed: the AFM-based axial tension, three-point bending, and nanoindentation tests. In a typical AFM-based axial tension test, one end of the nanofiber segment is fastened with adhesive onto a substrate (e.g., a silicon wafer), while the other end is tethered to the AFM tip [34]. The microscopic tensile force is exerted through the motion of the AFM tip. In the case of a micro three-point bending test, the fiber segment is clamped at two ends with adhesive onto a substrate with periodic gratings $[32,33,35]$. A lateral bending force is exerted through the AFM tip at the midspan of the nanofiber segment between neighboring supports. Furthermore, the axial modulus of nanofibers can also be mea- 
sured by means of nanoindentation, similarly to the characterization of thin films. After each test, a force vs displacement or deflection diagram is recorded and the axial modulus can be extracted. Moreover, the axial modulus of nanofibers can also be estimated by measuring the resonant frequencies of a pair of microcantilevers bridged by the nanofiber segments under consideration [36].

Nevertheless, the ultimate tensile strength and the strain to failure of nanofibers are arduous to determine using AFM. Indeed, the best way to characterize the mechanical properties of a nanofiber is by direct testing a single nanofiber segment on a microtension tester installed with a highresolution load cell. Newly available high-resolution microtensile testers such as the Nano Bionix and Nano UTM provided by MTS and microelectromechanical-systems(MEMS-) based microtensile testers [37,38] make this possible. Presently, full-range highly resolved stress-strain diagrams of nanofiber tension tests can be recorded. For instance, the Nano Bionix has been employed successfully for a uniaxial tension test of polycaprolactone fibers with diameters of $\sim 1 \mu \mathrm{m}$ [39]. A similar high-resolution microtensile tester was also produced and utilized recently to characterize the modulus and tensile strength of high-strength, hightoughness polyimide (PI) nanofibers with diameters of $\sim 300 \mathrm{~nm}$ [31]. In these tension tests, the tensile force is extremely low, ranging from a few millinewtons to a few micronewtons. Due to the very low tensile force, MEMSbased microtensile testers developed recently may provide an alternative for feasible and accurate measurements [37,38].

The above-referenced microtension tests have disclosed two unique behaviors of electrospun polymer nanofibers that are essentially different from those of their large-diameter and bulk counterparts. The first unique behavior is an abrupt increase in axial modulus displayed by a polymer nanofiber when its diameter falls below a certain value $[35,40,41]$. For instance, polystyrene nanofibers demonstrate a rapid increase of the axial modulus when their diameter is below $\sim 500 \mathrm{~nm}$; [40] while poly(2-acrylamido-2-methyl-1-propanesulfonic acid) nanofibers sharply increase their axial moduli from $\sim 300 \mathrm{MPa}$ at the diameter of $\sim 110 \mathrm{~nm}$ up to $\sim 2.0 \mathrm{GPa}$ at the diameter of $\sim 55 \mathrm{~nm}$ [40]. Surface tension may possibly contribute to such an increase in axial modulus according to a recent continuum mechanics calculation [28]; however, this explanation is insufficient when trying to understand such fiber behavior occurring below the critical diameter. Therefore, other potential factors need to be further explored. If one considers an electrostatic stretching in the mesojet during the electrospinning process, the observed remarkable size effect can be attributed to a gradual ordering of the microstructure as a function of fiber diameter $[35,41,42]$. As a matter of fact, recently x-ray diffraction has shown that there is only a mild monotonic increase in the crystallinity and orientation of the crystallites inside the polymer nanofibers as a function of fiber diameter [41]. As a result, such a mild increase would not be sufficient to account for the observed exceptional increase in the axial modulus. In addition, the above studies further indicated that the average size of crystallites $(\sim 4 \mathrm{~nm})$ was largely independent of the nanofiber diameter and therefore the crystallinity remained nearly constant in the range of fiber diameter under consideration. To

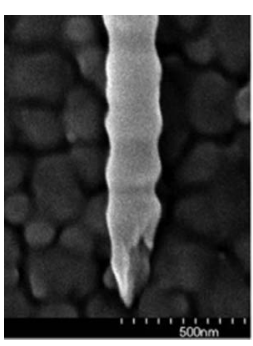

(a)

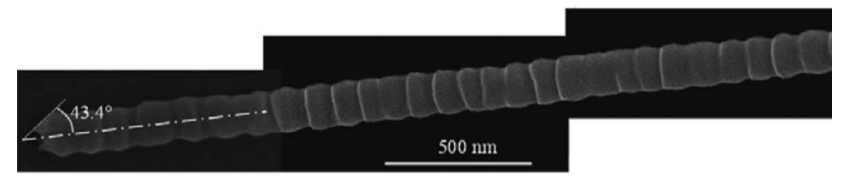

(d)
FIG. 1. (a), (b) Scanning electron microscope (SEM) images of surface morphology of as-electrospun PAN nanofibers after tensile breakage. The fiber breakage was induced by extrusion of a $45^{\circ}$ conic region. (c) SEM image of the fiber breakage due to the formation of voids. (d) SEM image of ripples formed on PAN nanofiber surfaces subjected to axial stretching $[37,38]$.

explore this size effect in the axial tensile modulus of polymer nanofibers, one promising physical mechanism [42] was proposed recently, based on the concept of the supramolecular structure of the amorphous phase, which consists of oriented fragments of the polymer chains. Such supramolecular structures might be formed due to the confinement of polymer nanofibers when the fiber diameter decreases below a certain value. According to this approach, a preliminary estimate was made in predicting the transition fiber diameter, below which the polymer nanofibers may exhibit a noticeable size effect in their axial modulus [41]. Yet this approach still leaves an open problem of the likely power-law growth of the axial modulus with decreasing fiber diameter below that certain value.

The second unique behavior is the surface rippling in compliant polymer nanofibers subjected to axial stretching. Such surface instability at the nanoscale was first detected in recent MEMS-based microtension tests of as-electrospun polyacrylonitrile (PAN) nanofibers [37,38]. In such tension tests, periodic ripples were detected on the fiber surfaces when the axial stretch was up to a certain level as shown in Fig. 1 [37,38]. It was also discovered during the singlenanofiber tension tests that the PAN nanofibers exhibited no clear softening, and the strain to failure (in the sense of engineering strain) was typically up to $60-130 \%$ for the electrospun PAN nanofibers with a diameter around $300-600 \mathrm{~nm}$. This strain is several fold greater than that obtained in PAN microfibers produced by drawing and dryjet wet spinning $[37,38]$. In addition, such surface ripples were also found in other electrospun polymer nanofibers, triggered by possible axial contraction after annealing. Figure 2 shows AFM images of the surface morphology of PI nanofibers produced by electrospinning and subsequent imi- 


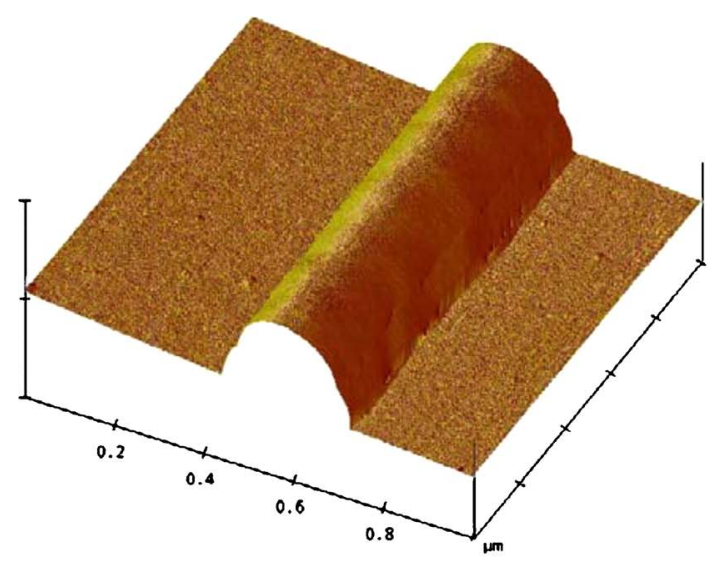

(a)

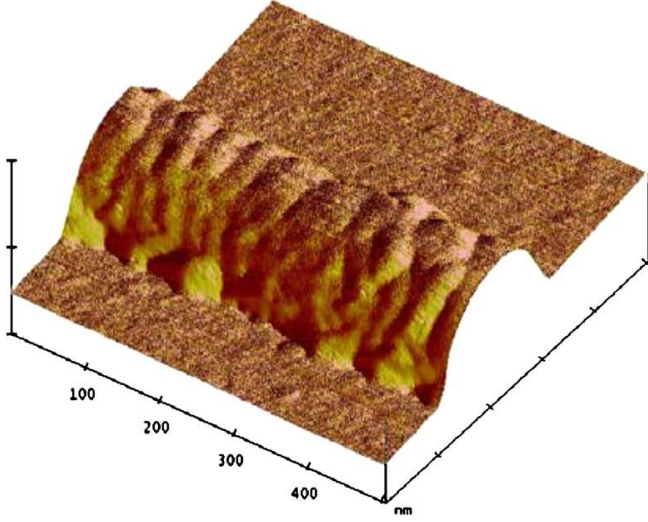

(b)

FIG. 2. (Color online) AFM images of surface morphology of (a) as-electrospun PI precursor nanofiber and (b) PI nanofiber after imidization (fiber diameter $\sim 250 \mathrm{~nm}$ ).

dization in our recent study. For smooth PI precursor nanofibers deposited on a silicon wafer in electrospinning, as shown in Fig. 2(a), after imidization in a nitrogen environment, surface ripples were detected. This rippling phenomenon may be attributed to the constrained axial shrinking induced in the imidization process.

As a matter of fact, the formation of surface ripples on PAN nanofibers is essentially due to a surface instability triggered by the combined effect of surface tension and nonlinear elasticity of the polymer nanofibers. Within the framework of nonlinear elasticity $[43,44]$, necking and surface instability of elastic bars subjected to axial stretching have been well studied, where the effect of surface tension or energy was excluded due to the relatively large diameter and high elastic modulus of the rods. However, at the nanoscale, surface tension may play a crucial role in triggering the surface rippling in compliant ultrathin nanofibers.

Therefore, in this study we consider the evolution mechanism of surface rippling in compliant polymer nanofibers subjected to large axial stretching. As a first approach, we propose a simple 1D nonlinear elastic model to examine the combined effect of surface tension and nonlinear elasticity on ripple formation. The governing equation is established through linear perturbation of the static equilibrium state of a prestretched compliant nanofiber. Without loss of generality in capturing the main features, we simplify the compliant nanofiber as an incompressible, isotropically hyperelastic Mooney-Rivlin solid. The critical stretch in triggering the surface rippling and the corresponding ripple wavelength are determined in terms of surface tension, elastic properties, and fiber radius. Numerical examples are demonstrated to examine these dependencies. Furthermore, the present model will be further used to explain the experimental results obtained in recent single-nanofiber tension tests. Then the conclusions and potential applications of the present study are summarized.

\section{MODEL DEVELOPMENT}

Consider a compliant polymer nanofiber as an infinitely long and thin rod made of an incompressible, isotropically hyperelastic Mooney-Rivlin solid. In reality, polymer chains inside a polymer fiber may have some extent of preferred orientation and crystallinity owing to the electrostatic stretching in an electrospinning process. For the undisturbed stretch-free state, the imaginary configuration of the nanofiber (with surface tension ignored) is assumed to be a perfectly circular cylinder of radius $R_{0}$. For the convenience of our discussion afterward, three configurations are adopted to describe the motion of a material point inside the fiber, i.e., undisturbed stretch-free (with surface tension ignored), prestretched (with surface tension), and current configuration (with surface ripples), respectively. The corresponding coordinates of the material point are denoted by $(R, \Theta, Z)$, $(r, \theta, z)$, and $(\widetilde{r}, \tilde{\theta}, \tilde{z})$, respectively. In the following, we first derive the solution for the prestretched state, and then establish the governing equation of a surface rippling through linear perturbation of the prestretched state.

\section{A. Thin solid fibers under axial prestretching}

When subjected to axial uniform stretching, the axisymmetric deformation of a prestretched fiber can be expressed as

$$
\begin{gathered}
r=\lambda_{1} R \quad\left(0 \leqslant R \leqslant R_{0}\right), \quad \theta=\Theta \quad(0 \leqslant \Theta \leqslant 2 \pi), \\
z=\lambda_{3} Z \quad(-\infty \leqslant Z \leqslant+\infty),
\end{gathered}
$$

where $\lambda_{1}$ and $\lambda_{3}$ are constants, corresponding to the transverse and longitudinal stretches, respectively. The deformation gradient of the above deformation is

$$
\mathbf{F}=\left[\begin{array}{ccc}
\partial r / \partial R & (1 / R) \partial r / \partial \Theta & \partial r / \partial Z \\
r \partial \theta / \partial R & (r / R) \partial \theta / \partial \Theta & r \partial \theta / \partial Z \\
\partial z / \partial R & (1 / R) \partial z / \partial \Theta & \partial z / \partial Z
\end{array}\right]=\left[\begin{array}{ccc}
\lambda_{1} & 0 & 0 \\
0 & \lambda_{1} & 0 \\
0 & 0 & \lambda_{3}
\end{array}\right] .
$$

The material incompressibility of the polymer fiber requires $\lambda_{1}$ and $\lambda_{3}$ satisfying 


$$
\lambda_{1}^{2} \lambda_{3}=1 \text {. }
$$

The resulting left Cauchy-Green tensor $\mathbf{B}$ and its inverse are, respectively,

$$
\mathbf{B}=\mathbf{F F}^{T}=\operatorname{diag}\left[\lambda_{1}^{2}, \lambda_{1}^{2}, \lambda_{3}^{2}\right], \quad \mathbf{B}^{-1}=\operatorname{diag}\left[\lambda_{1}^{-2}, \lambda_{1}^{-2}, \lambda_{3}^{-2}\right] .
$$

The scalar invariants of $\mathbf{B}$ are

$$
I_{1}=2 \lambda_{1}^{2}+\lambda_{3}^{2}=2 \lambda_{3}^{-1}+\lambda_{3}^{2}, \quad I_{2}=2 \lambda_{3}+\lambda_{3}^{-2}, \quad I_{3}=1 .
$$

The constitutive law of the compliant polymer nanofiber is assumed to obey the equation for a general incompressible, isotropically hyperelastic Mooney-Rivlin solid, which can be expressed in terms of the Cauchy stress tensor vs $\mathbf{B}$ :

$$
\mathbf{T}=-p \mathbf{I}+2 c_{1} \mathbf{B}+c_{2} \mathbf{B}^{-1},
$$

where $p$ is the hydrostatic pressure, and $c_{1}$ and $c_{2}$ are two independent material parameters. In the special case of $c_{2}$ $=0$ and $c_{1}$ half the shear modulus, a material satisfying the constitutive relation (6) is called a neo-Hookean solid. In terms of stress components, it reads

$$
\begin{gathered}
T_{r r}=T_{\theta \theta}=-p+2 c_{1} \lambda_{1}^{2}+c_{2} \lambda_{1}^{-2}=-p+2 c_{1} \lambda_{3}^{-1}+c_{2} \lambda_{3}, \\
T_{z z}=-p+2 c_{1} \lambda_{3}^{2}+c_{2} \lambda_{1}^{4}=-p+2 c_{1} \lambda_{3}^{2}+c_{2} \lambda_{3}^{-2}, \\
T_{r \theta}=T_{r z}=T_{\theta z}=0 .
\end{gathered}
$$

Furthermore, in spatial coordinates, the equilibrium equations of the axisymmetrically deformed fiber can be written as

$$
\begin{gathered}
\frac{\partial T_{r r}}{\partial r}+\frac{T_{r r}-T_{\theta \theta}}{r}=0, \\
\frac{\partial T_{\theta \theta}}{\partial \theta}=0, \\
\frac{\partial T_{z z}}{\partial z}=0 .
\end{gathered}
$$

In the above, two traction boundary conditions (BCs) have been triggered. At the fiber surface, the surface tension leads to uniform compression radically, i.e.,

$$
T_{r r}=-\gamma / r_{0},
$$

where $\gamma(\mathrm{N} / \mathrm{m})$ is the surface tension of the amorphous polymer fiber, which is assumed to be independent of the fiber radius and applied axial stretch in this study, and $r_{0}$ is the fiber radius in the current configuration after deformation. In addition, along the fiber axis, axial force equilibrium requires

$$
P=2 \pi \int_{0}^{r_{0}} r T_{z z} d r+2 \pi r_{0} \gamma
$$

where $P$ is the axial tensile force resultant. The relationship between $P$ and the axial stretch $\lambda_{3}$ of the fiber can be determined by solving (10)-(12) under traction conditions (13) and (14) such that [28]

$$
P=\pi r_{0}^{2}\left(\lambda_{3}^{2}-\lambda_{3}^{-1}\right)\left(2 c_{1}-c_{2} \lambda_{3}^{-1}\right)+\pi r_{0} \gamma
$$

The above relation can also be expressed in terms of the radius of a stretch-free fiber (with surface tension ignored) by applying the deformation relation $r_{0}=\lambda_{1} R_{0}=R_{0} \lambda_{3}^{-1 / 2}$ :

$$
P=\pi R_{0}^{2}\left(\lambda_{3}-\lambda_{3}^{-2}\right)\left(2 c_{1}-c_{2} \lambda_{3}^{-1}\right)+\pi R_{0} \gamma \lambda_{3}^{-1 / 2} .
$$

\section{B. Rippling of thin polymer fibers under axial stretching}

Let us now consider the 1D rod equation for a small disturbance superimposed on the prestretched state of the polymer nanofiber in the condition of axisymmetric deformation. For small disturbance, the coordinates of a material point in the current configuration can be expressed as

$$
\begin{gathered}
\tilde{r}=\left[\lambda_{1}+f(Z)\right] R \quad\left(0 \leqslant R \leqslant R_{0}\right), \quad \tilde{\theta}=\Theta \quad(0 \leqslant \Theta \leqslant 2 \pi), \\
\tilde{z}=\lambda_{3} Z+g(Z) \quad(-\infty \leqslant Z \leqslant+\infty),
\end{gathered}
$$

where $f(Z)$ and $g(Z)$ are two small disturbance functions satisfying the BCs (13) and (14). The corresponding deformation gradient matrix $\widetilde{\mathbf{F}}$ and the left Cauchy-Green tensor $\widetilde{\mathbf{B}}$ can be determined as

$$
\begin{gathered}
\tilde{\mathbf{F}}=\left[\begin{array}{ccc}
\lambda_{1}+f & 0 & f_{Z} R \\
0 & \lambda_{1}+f & 0 \\
0 & 0 & \lambda_{3}+g_{Z}
\end{array}\right], \\
\widetilde{\mathbf{B}}=\tilde{\mathbf{F}} \widetilde{\mathbf{F}}^{T}=\left[\begin{array}{ccc}
\left(\lambda_{1}+f\right)^{2}+\left(f_{Z} R\right)^{2} & 0 & f_{Z} R\left(\lambda_{3}+g_{Z}\right) \\
0 & \left(\lambda_{1}+f\right)^{2} & 0 \\
f_{Z} R\left(\lambda_{3}+g_{Z}\right) & 0 & \left(\lambda_{3}+g_{Z}\right)^{2}
\end{array}\right] .
\end{gathered}
$$

As a result, the three scalar invariants of $\widetilde{B}$ are

$$
\begin{gathered}
I_{1}=2\left(\lambda_{1}+f\right)^{2}+\left(f_{Z} R\right)^{2}+\left(\lambda_{3}+g_{Z}\right)^{2}, \\
I_{2}=\left(\lambda_{1}+f\right)^{2}\left[\left(\lambda_{1}+f\right)^{2}+\left(f_{Z} R\right)^{2}+2\left(\lambda_{3}+g_{Z}\right)^{2}\right], \\
I_{3}=\left(\lambda_{1}+f\right)^{4}\left(\lambda_{3}+g_{Z}\right)^{2} .
\end{gathered}
$$

Material incompressibility of the polymer fiber (22) leads to

$$
\left(\lambda_{1}+f\right)^{2}\left(\lambda_{3}+g_{Z}\right)=1 .
$$

Relation (23) implies that $f$ and $g$ are correlated, and they can be determined through the variational principle applied onto the potential energy of the entire fiber. For the incompressible, isotropically hyperelastic Mooney-Rivlin solid under consideration, the corresponding strain energy density is defined as [45]

$$
e=c_{1}\left(I_{1}-3\right)+c_{2}\left(I_{2}-3\right),
$$

where $I_{1}$ and $I_{2}$ are, respectively, the first and second invariants of the left Cauchy-Green tensor $\widetilde{\mathbf{B}}$ as given in (20) and (21), and $c_{1}$ and $c_{2}$ are two material parameters of the Mooney-Rivlin solid. Thus, the potential energy functional $\Pi$ of the compliant nanofiber subjected to axial stretching is 


$$
\begin{aligned}
\Pi= & \pi R_{0}^{2} \int_{L} e d Z+2 \pi R_{0} \gamma \int_{L}\left(\lambda_{1}+f\right)\left(\lambda_{3}+g_{Z}\right) d Z \\
& -P \int_{L}\left(\lambda_{3}+g_{Z}-1\right) d Z .
\end{aligned}
$$

The three terms in (25) are the contributions due to elastic strain energy, surface energy, and work done by the axial tensile force $P$, respectively. In addition, in relation (25) material incompressibility has been taken into account, and the integration with respect to $Z$ runs over the entire length of the fiber segment.

By substituting (20)-(24) into (25) and then triggering the variational principle [27] on (25), a second-order nonlinear ordinary differential equation (ODE) can be obtained as

$$
\begin{aligned}
R_{0}^{2}(1 & \left.+c_{2} / c_{1} \lambda_{1}^{2}\right) f_{Z Z}-\left\{6 c_{2} / c_{1} \lambda_{1}^{2}+2+2 \gamma /\left(c_{1} R_{0}\right) \lambda_{1}^{-3}\right. \\
& \left.-3\left[P /\left(c_{1} \pi R_{0}^{2}\right)-2 c_{2} / c_{1}\right] \lambda_{1}^{-4}+10 \lambda_{1}^{-6}\right\} f \\
& -\left\{2 c_{2} / c_{1} \lambda_{1}^{3}+2 \lambda_{1}-\gamma /\left(c_{1} R_{0}\right) \lambda_{1}^{-2}+\left[P /\left(c_{1} \pi R_{0}^{2}\right)\right.\right. \\
& \left.\left.-2 c_{2} / c_{1}\right] \lambda_{1}^{-3}-2 \lambda_{1}^{-5}\right\}=0 .
\end{aligned}
$$

In the case of linear perturbation as used for the study of rippling initiation, $f$ in (26) is a small disturbance from the prestretched state. By eliminating the higher-order terms of $f$ and $f_{Z}$ in (26), a second-order linear ODE can be extracted which governs the rippling initiation, i.e.,

$$
A f_{Z Z}+B f+C=0 .
$$

Here, the coefficients $A, B$, and $C$ in (27) are related to the surface energy, elastic properties, fiber geometry (radius), and applied prestretch such that

$$
\begin{gathered}
A=R_{0}^{2}\left(1+c_{2} / c_{1} \lambda_{3}^{-1}\right), \\
B=-\left[2+6 c_{2} / c_{1} \lambda_{3}^{-1}+2 \gamma /\left(c_{1} R_{0}\right) \lambda_{3}^{3 / 2}-3 P /\left(c_{1} \pi R_{0}^{2}\right) \lambda_{3}^{2}\right. \\
\left.+6 c_{2} / c_{1} \lambda_{3}^{2}+10 \lambda_{3}^{3}\right], \\
C=-\left[2 c_{2} / c_{1} \lambda_{3}^{-3 / 2}+2 \lambda_{3}^{-1 / 2}-\gamma /\left(c_{1} R_{0}\right) \lambda_{3}+P /\left(c_{1} \pi R_{0}^{2}\right) \lambda_{3}^{3 / 2}\right. \\
\left.-2 c_{2} / c_{1} \lambda_{3}^{3 / 2}-2 \lambda_{3}^{5 / 2}\right],
\end{gathered}
$$

where material incompressibility (3) has been implied.

As a matter of fact, surface rippling in a compliant nanofiber implies the existence of periodical solution to Eq. (27). This yields the corresponding characteristic equation:

$$
A f_{Z Z}+B f=0 \text {. }
$$

Assume the periodic solution to (31) in the following form:

$$
f(Z)=A_{0} \exp (i k Z),
$$

where $A_{0}$ is the complex amplitude of surface disturbance, and $k$ is the wave number. Therefore, substituting (32) into (31) leads to

$$
k=\sqrt{B / A},
$$

which is a positive number for a physically meaningful rippling surface. As a result, the condition for surface rippling of compliant rubbery polymer nanofibers is

$$
B / A>0,
$$

and the corresponding ripple wavelength can be expressed as

$$
\lambda=2 \pi / k \text {. }
$$

Consequently, relation (34) as well as (15), (28), and (29) determine the rippling condition of compliant polymer nanofibers subjected to axial stretching.

\section{NUMERICAL EXAMPLES OF RIPPLING DEPENDENCY AND DISCUSSIONS}

\section{A. Critical condition of surface rippling in compliant polymer nanofibers}

Based on the rippling condition (34), the critical axial stretch, beyond which rippling happens, can be determined by letting

$$
B=0 \text {, }
$$

i.e.,

$$
\begin{aligned}
2+ & 6 c_{2} / c_{1} \lambda_{3}^{-1}+2 \gamma /\left(c_{1} R_{0}\right) \lambda_{3}^{3 / 2}-3 P /\left(c_{1} \pi R_{0}^{2}\right) \lambda_{3}^{2} \\
& +6 c_{2} / c_{1} \lambda_{3}^{2}+10 \lambda_{3}^{3}=0,
\end{aligned}
$$

where $P$ is the axial force resultant given in (15). It can be proved that Eq. (37) may have one, two, or no real roots depending upon the combined effect of surface tension, elastic properties, and fiber radius. For typical rubbery hyperelastic materials, it guarantees that $A>0$ in (28). Thus, it is expected that for sufficient low loading rate and large fiber diameter, compliant polymer fibers may have only a single necking that happens at $B \leqslant 0$ in (29). In such a case, the final fiber breakage takes place at the necking locus as commonly observed in uniaxial tension tests of polymer fibers. In addition, the prestretched polymer fiber under consideration can be understood as follows: the fiber is deformed to a given prestretch in a relatively short time interval and then this constant prestretch is sustained.

\section{B. Critical radii for rippling in compliant polymer nanofibers}

Let us further consider the surface elastic instability of compliant polymer nanofibers in the stretch-free state (i.e., $\lambda_{3}=1$ ). By setting $\lambda_{3}=1$ in relations (16) and (37), the critical condition $B=0$ leads to the critical fiber radius $R_{C}$ :

$$
R_{C}=\gamma /\left[12\left(c_{1}+c_{2}\right)\right] .
$$

Correspondingly, the derivative of $B$ in (29) with respect to $R_{0}$ in the stretch-free state gives

$$
\left.\frac{d B}{d R_{0}}\right|_{\lambda_{3}=1}=-\frac{\gamma}{c_{1} R_{0}^{2}}<0 .
$$

The above relation indicates that for $R_{0}<R_{C}, B>0$ holds in the stretch-free state $\left(\lambda_{3}=1\right)$, i.e., below the critical fiber radius $R_{C}$, rippling can be triggered even without external stretch. In this case, the compliant fibers are intrinsically unstable and therefore cannot be physically produced in reality according to this model. Thus, an initially stable polymer 


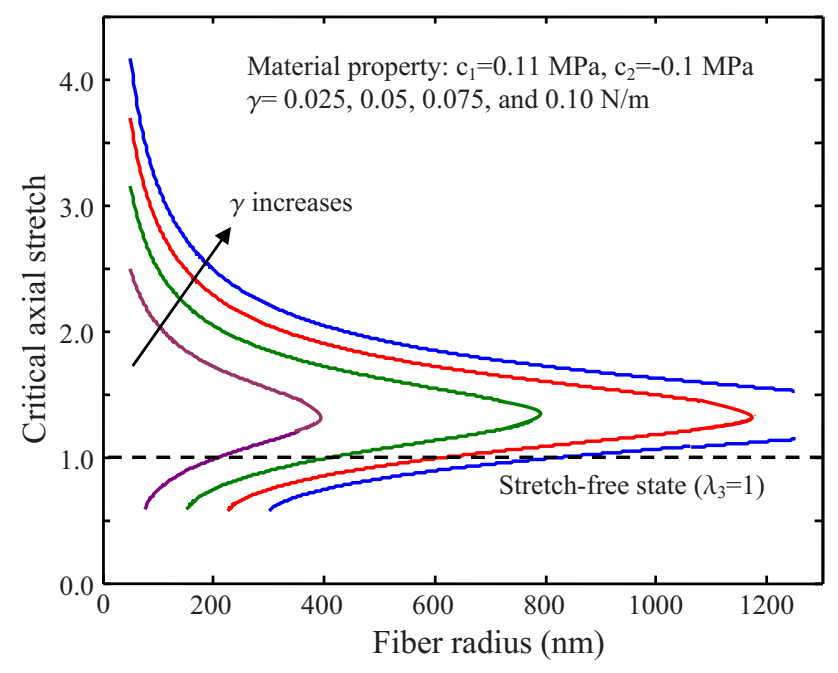

FIG. 3. (Color online) Variation of the upper and lower critical stretches vs the fiber radius for cases of varying surface tension.

nanofiber at the stretch-free state $\left(\lambda_{3}=1\right)$ must satisfy $B<0$, i.e., $R>R_{C}$ according to (38) and (39). Consequently, if solving $R_{0}$ with the condition $B=0$ in (29) and then considering the stationary point satisfying $d R_{0} / d \lambda_{3}=0$, one may obtain the upper limit radius of surface rippling. Beyond this radius, rippling could not happen.

It needs to be mentioned that, for the convenience of the above derivation, we have constrained the material in this study to be a general incompressible, isotropically hyperelastic Mooney-Rivlin solid. Such a material model is a feasible approach to handle many rubbery polymers analytically. For as-electrospun PAN nanofibers, our recent experiments indicated that these ultrathin fibers were extremely compliant with very large strain to failure (around 150\%) and very large residual plastic strain compared to their counterparts of microextrusion fibers and cast bulk polymers. Nevertheless, in the study of surface rippling, the Mooney-Rivlin material model provides a good approximation by taking into account this large axial stretch and capturing the main features during the rippling process.

Hereafter, we demonstrate some numerical examples to examine the rippling dependencies upon fiber radius and prestretch. The elastic constants of the polymer nanofiber, $c_{1}$ and $c_{2}$, are selected in the range of typical vulcanized rubber compounds [46] such that $c_{1}=0.1-0.31 \mathrm{MPa}, c_{2}$ $=-0.1 \mathrm{MPa}$, and the surface tension $\gamma=0.025-0.1 \mathrm{~N} / \mathrm{m}$. Figure 3 shows the variation of the upper and lower critical axial stretches needed to trigger the rippling for different fiber radii with varying surface tension. By examining the parameters $A$ and $B$ in (28) and (29), it is found that coefficient $A$ is always positive, while $B$ is a convex function with respect to the axial stretch $\lambda_{3}$. As a matter of fact, the physically meaningful $\lambda_{3}$ requires $B$ to be positive, and this leads to the upper and lower critical axial stretches for a given fiber radius and given material properties, as illustrated in Fig. 3. From Fig. 3, one can observe that the upper critical axial stretch decreases with either increasing fiber radius or decreasing fiber surface tension. Meanwhile, the lower critical axial stretch increases with increasing either the fiber

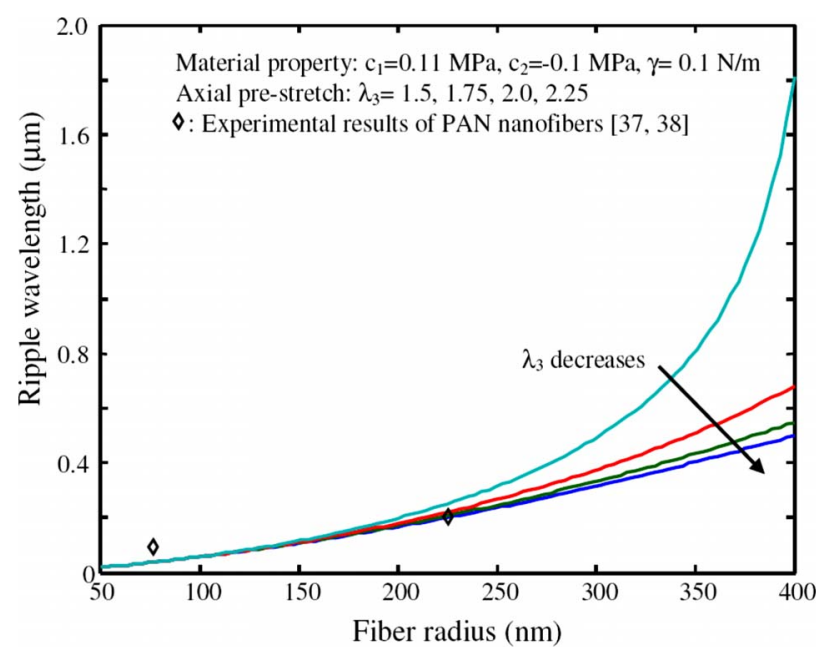

FIG. 4. (Color online) Variation of the ripple wavelength vs the fiber radius for cases of varying prestretch. (Inserted symbols $\diamond$ are the experimental results based on single-fiber tension test $[37,38]$.)

radius or the surface tension. In addition, Fig. 3 further implies that, for a rubbery nanofiber with diameter below a certain value, the fiber may be naturally unstable as predicted in (38). It can be observed in Fig. 3 that the upper and lower critical axial stretches may overlap at a certain fiber radius. Beyond this critical radius, surface rippling could not happen. As a result, for a given axial prestretch between the upper and lower critical limits, surface rippling may happen according to the present rippling model. The upper and lower limits of critical stretch also determine the range of ripple wavelength of polymer nanofiber rippling under axial stretching.

Furthermore, Fig. 4 indicates the variation of the ripple wavelength as a function of fiber radius with varying prestretch between the two upper and lower limits of critical stretch. One can see that the ripple wavelength increases with the increase of either fiber radius or prestretch for given elastic properties and surface tension of the fiber material. For the purpose of comparison between the present model and experimental measurements, the ripple wavelengths and corresponding fiber radii of two types of as-electrospun PAN nanofibers available in the literature $[37,38]$ are plotted in Fig. 4. One can see that the ripple wavelength of the PAN nanofibers as detected in single-fiber tension tests $[37,38]$ (see Fig. 1) are located within the wavelength range as predicted in the present model. Indeed, the present continuum mechanics model reveals the rippling mechanism in polymer nanofibers, i.e., the surface rippling phenomenon can be approached within the framework of surface instability of hyperelastic solids. Moreover, the present model can be further refined to take into account other potential factors such as plasticity, viscoelasticity, material compressibility, elastic anisotropy, and dynamics.

\section{CONCLUDING REMARKS}

A 1D phenomenological nonlinear elastic model has been developed which can describe the mechanism of surface rip- 
pling in compliant polymer nanofibers subjected to large axial stretch. Based on the assumption of a general incompressible, isotropically hyperelastic Mooney-Rivlin solid, the present model presented reasonable scaling properties of rippling dependencies upon the surface tension, elastic properties, fiber radius, and axial pre-stretch. It can be seen in Fig. 3 that, for polymer fibers of large diameter, rippling may not happen since the upper and lower critical stretches tend to intersect at a certain fiber diameter. In this case, the tensile failure of the polymer fiber is necking-related breakage, as commonly observed in conventional polymer fibers. In addition, the predictions given by the present model can be validated by experimental results obtained in recent single-fiber tension tests.

The emphasis should be brought to the point that the actual ripples formed in electrospun polymer nanofibers have significant plastic deformation in a monotonic axial tension test. Such plastic deformation makes it possible to detect the surface ripples by means of a scanning electron microscope or AFM after unloading. The explanation of such an effect on the rippling initiation remains open. Therefore, further refinement of the rippling model is desired, and needs to include the material plasticity, viscoelasticity, compressibility, and elastic anisotropy. A more detailed approach is expected to provide in-depth understanding of the surface rippling in ultrathin compliant polymer fibers. In addition, pure nonlinear numerical methods (e.g., the finite-element method) can be adopted to capture the entire evolution process of surface rippling and relevant effects of the control parameters. Consequently, the present model discloses the means for developing nonlinear dynamic models important for the analysis of wave propagation in polymer nanofibers that are potentially required for the development of nanofiber transducers, sensors, and other applications.

\section{ACKNOWLEDGMENTS}

Partial support of this work by the NSF, AFOSR, and ARL is gratefully acknowledged. The work at NDSU was supported by a Faculty Startup Grant.
[1] D. H. Reneker and I. Chun, Nanotechnology 7, 216 (1996).

[2] Y. Dzenis, Science 304, 1917 (2004).

[3] P. Gibson, H. Schreuder-Gibson, and D. Rivin, Colloids Surf., A 187, 469 (2001).

[4] H. Schreuder-Gibson, P. Gibson, K. Senecal, M. Sennett, J. Walker, W. Yeomans, D. Ziegler, and P. P. Tsai, J. Adv. Mater. 34, 44 (2002).

[5] P. P. Tsai, H. Schreuder-Gibson, and P. Gibson, J. Electrost. 54, 333 (2002).

[6] C. Shin and G. G. Chase, J. Dispersion Sci. Technol. 27, 517 (2006).

[7] J. S. Kim and D. H. Reneker, Polym. Compos. 20, 124 (1999).

[8] H. Q. Hou and D. H. Reneker, Adv. Mater. (Weinheim, Ger.) 16, 69 (2004).

[9] C. Lai, Q. H. Guo, X. F. Wu, D. H. Reneker, and H. Hou, Nanotechnology 19, 195303 (2008).

[10] Y. Wang, S. Serrano, and J. J. Santiago-Aviles, Synth. Met. 138, 423 (2003).

[11] S. Y. Gu, J. Ren, and G. J. Vancso, Eur. Polym. J. 41, 2559 (2005).

[12] W. J. Li, C. T. Laurencin, E. J. Caterson, R. S. Tuan, and F. K. Ko, J. Biomed. Mater. Res. 60, 613 (2002).

[13] H. Yoshimoto, Y. M. Shin, H. Terai, and J. P. Vacanti, Biomaterials 24, 2077 (2003).

[14] S. Y. Chew, Y. Wen, Y. Dzenis, and K. W. Leong, Curr. Pharm. Des. 12, 4751 (2006).

[15] K. Sawicka, P. Gouma, and S. Simon, Sens. Actuators B 108, 585 (2005).

[16] Y. Wang, I. Ramos, and J. J. Santiago-Aviles, IEEE Sens. J. 7, 1347 (2007).

[17] K. H. Lee, D. J. Kim, B. G. Min, and S. H. Lee, Biomed. Microdevices 9, 435 (2007).

[18] W. E. Teo and S. Ramakrishna, Nanotechnology 17, R89 (2006).

[19] Z. M. Huang, Y. Z. Zhang, M. Kotaki, and S. Ramakrishna,
Compos. Sci. Technol. 63, 2223 (2003).

[20] D. Li and Y. N. Xia, Adv. Mater. (Weinheim, Ger.) 16, 1151 (2004).

[21] S. G. Kumbar, S. P. Nubavarapu, J. James, M. V. Hogan and T. Laurencin, Recent Patents Biomed. Eng. 1, 68 (2008).

[22] E. P. S. Tan and C. T. Lim, Compos. Sci. Technol. 66, 1102 (2006).

[23] D. H. Reneker, A. L. Yarin, E. Zussman, and H. Xu, Adv Appl. Mech. 41, 43 (2007).

[24] R. Ramaseshan, S. Sundarrajan, R. Jose, and S. Ramakrishna, J. Appl. Phys. 102, 111101 (2007).

[25] X. F. Wu and Y. A. Dzenis, J. Appl. Phys. 98, 093501 (2005).

[26] X. F. Wu and Y. A. Dzenis, J. Appl. Phys. 100, 124318 (2006).

[27] X. F. Wu and Y. A. Dzenis, Acta Mech. 185, 215 (2006).

[28] X. F. Wu and Y. A. Dzenis, J. Appl. Phys. 102, 044306 (2007).

[29] X. F. Wu and Y. A. Dzenis, Nanotechnology 18, 285702 (2007).

[30] X. F. Wu and Y. A. Dzenis, J. Phys. D 40, 4276 (2007).

[31] F. Chen, X. W. Peng, T. T. Li, S. L. Chen, X. F. Wu, D. H Reneker, and H. Q. Hou, J. Phys. D 41, 025308 (2008).

[32] E. P. S. Tan and C. T. Lim, Rev. Sci. Instrum. 75, 2581 (2004).

[33] E. P. S. Tan, C. N. Goh, C. H. Sow, and C. T. Lim, Appl. Phys. Lett. 86, 073115 (2005).

[34] E. Zussman, M. Burman, A. L. Yarin, R. Khalfin, and Y. Cohen, J. Polym. Sci., Part B: Polym. Phys. 44, 1482 (2006).

[35] M. K. Shin, S. I. Kim, S. J. Kim, S. K. Kim, H. Lee, and G. M. Spinks, Appl. Phys. Lett. 89, 231929 (2006).

[36] P. A. Yuya, Y. K. Wen, J. A. Turner, Y. A. Dzenis, and Z. Li, Appl. Phys. Lett. 90, 111909 (2007).

[37] M. Naraghi, I. Chasiotis, H. Kahn, Y. K. Wen, and Y. Dzenis, Appl. Phys. Lett. 91, 151901 (2007).

[38] M. Naraghi, I. Chasiotis, H. Kahn, Y. Wen, and Y. Dzenis, Rev. Sci. Instrum. 78, 085108 (2007).

[39] E. P. S. Tan, S. Y. Ng, and C. T. Lim, Biomaterials 26, 1453 (2005). 
[40] Y. Ji, B. Q. Li, S. R. Ge, J. C. Sokolov, and M. H. Rafailovich, Langmuir 22, 1321 (2006).

[41] A. Arinstein, M. Burman, O. Gendelman, and E. Zussman, Nat. Nanotechnol. 2, 59 (2007).

[42] C. T. Lim, E. P. S. Tan, and S. Y. Ng, Appl. Phys. Lett. 92, 141908 (2008).

[43] B. D. Coleman, Arch. Ration. Mech. Anal. 83, 115 (1983).
[44] H. H. Dai and Z. Cai, Acta Mech. 139, 201 (2000).

[45] Y. B. Fu and R. W. Ogden, Nonlinear Elasticity: Theory and Applications (Cambridge University Press, Cambridge, U. K., 2001).

[46] L. T. G. Trelorar, The Physics of Rubber Elasticity (Clarendon, Oxford, 1975). 